\title{
Dynamic Stability Safety Evaluation of Consequent Rock Slopes
}

\author{
Shi YAN ${ }^{1, a,{ }^{*}, \text { Haitao DU }}{ }^{2, b}$, Yaoyao $\mathrm{CHEN}^{1, c}$, Xuenan WANG ${ }^{1, d}$ \\ ${ }^{1}$ School of Civil Engineering, Shenyang Jianzhu University, Shenyang, Liaoning, China \\ ${ }^{2}$ Liaoning Electric Power Survey \& Design Institute, Shenyang, China \\ acesyan@sjzu.edu.cn, bduhaitao0330@126.com, c1837738588@qq.com, d364120715@qq.com \\ ${ }^{*}$ Corresponding author
}

Keywords: Consequent rock slope; Dynamic stability; FLAC3D; Instability mechanism; Instability criterion; Strength reduction method; Safety factor.

Abstract. The dynamic stability issue of consequent rock slopes has been paid wide attention because this kind of slope is more likely to form a buckling failure under seismic loading. In this paper, in-depth researches focused on the dynamic stability problem of consequent rock slope have been launched by using the FLAC3D software. Based on the numerical method, the slope instability mode and instability criterion under earthquake motions are proposed. Four seismic waves are adopted, respectively, to evaluate the dynamic stability of consequent rock slope by using the dynamic strength reduction method (DSRM), and the dynamic safety factor of slope under the frequently occurred earthquake is calculated.

\section{Introduction}

Consequent rock slope (CRS) is usually defined as the stratified rock mass slope whose slope free face has the similar dip direction with the rock stratum [1]. The instable CRS sliding that most frequently occurred among mountain roads and near building sites might generate serious geological disaster, bringing huge loss lives and properties. The probability of rock slope failure induced by geological disasters (including landslide and collapse) will greatly increase with the increasingly frequent earthquake disasters. Therefore, the CRS stability analysis is of great importance to investigate the mechanism of the landslide and collapse and take appropriate measures to prevent it happening or mitigate the geological disaster loss. However, the most of researches focused on the static stability analysis of high and steep slopes, but few on the CRS dynamic stability issues $[2,3]$. The paper aims at proposing a numerical method to analyze the dynamic stability performances, and evaluating the dynamic safety of a CRS under earthquakes by using the FLAC3D software.

\section{The CRS Dynamic Stability Analysis Method}

FLAC3D Software. In recent years, solving complicated slope issues through numerical analysis methods has become a trend for a great meaningful but huge cost research work. The continuum mechanics for finite element analysis (FEA) software FLAC3D is an excellent representative of geotechnical numerical analysis software which is developed by Itasca consulting companies in the United States and highly renowned in the academia and industry of civil engineering. FLAC3D is named from the use of Fast Lagrangian Analysis of Continua, which adopts the hybrid discrete method, the finite difference method and the dynamic relaxation method. 
Strength Reduction Method (SRM). The concept of strength reduction method was firstly put forward and applied to the slope stability analysis by Zienkiewize in 1975 [4]. In the SRM, the slope stability safety factor can be defined according to the reduction degree of geotechnical shear strength as the slope just reached the critical failure state [5]. Namely the safety factor is the ratio of the actual shear strength of rock and soil mass to the reduced shear strength of critical damage:

$$
\begin{aligned}
& c_{t}=c / F_{t} \\
& \varphi_{t}=\tan ^{-1}\left(\tan \varphi / F_{t}\right)
\end{aligned}
$$

where $c$ and $\varphi$ are the cohesive force and the internal friction angle of rock and soil mass, respectively. $c_{t}$ and $\varphi_{t}$ are the cohesive force and angle of internal friction after reduction, respectively. $F_{t}$ is the reduction factor. The key point of the SRM is to use the above formulas to adjust the value of rock mass strength, and then carry on the numerical analysis of the rock mass slope. By constantly increasing reduction factor $F_{t}$, the calculation is repeatedly performed until the slope reaches the state of critical destruction, and this reduction factor is defined as the safety factor of the slope stability $F_{s}$. Generally the critical failure state can be determined through the convergence of numerical calculation, the displacement mutability of the characteristic point and the connectivity of the plastic zone.

Compared with the traditional limit equilibrium method, the SRM has the following advantages in evaluating the slope stability:

(1) It is possible to simulate the slope with complex topography and geological structure.

(2) The influence of the material constitutive relation and the deformation is taken into account when calculating the slope stability.

(3) When calculating the safety factor, the slope instability mechanism can be explained well and the location and shape of slope sliding surface can be determined exactly without any assumption in advance.

(4) Combined action between rock mass and supporting structure can be simulated.

\section{Theoretical Analysis on Dynamic Instability Failure Mechanism}

In order to correctly evaluate the dynamic stability of the CRS, the dynamic instability failure mode of the slope and the corresponding buckling failure mechanism must be firstly determined. The critical instability state of the slope will not be reasonably judged and the dynamic stability will not be exactly evaluated until the dynamic failure characteristic is determined. In general, the CRS generating shear slip failure is most common and destructive. Therefore, the instability failure mechanism of this kind of CRS is mainly studied in this section.

Mao and $\mathrm{Hu}$ believed that the landslide of a CRS is mainly caused by the oscillation induced by earthquake in the slope $[6,7]$. They summarized the dynamic process of deformation, failure movement of consequent slope under seismic loads as three effects: the progressive failure effect, set-out slide effect and acceleration effect.

Progressive Failure Effect. The progressive failure effect refers to the phenomenon that the slope rock mass gradually forms material failure with the increase of the earthquake cycle times. In another word, the accumulative effect in an earthquake causes the failure of the slope rock mass.

Slope rock mass can generate inertial forces under seismic loads in both the horizontal and vertical directions. Because there are various kinds of cracks and weak structural planes in rock mass, the additional stress generated by the inertial force can produce stress concentration at the crack tip. When the shear or tensile strength of the rock mass is not strong enough to bear the shear or tensile stress produced by the inertia force, the local shear or tensile failure will first take place in 
the cracks of the slope, prompting the further development of the cracks. With the expansion of the cracks, a through failure plane will develop eventually. At this point, the upper rock mass may slide along the failure plane under seismic loads. This above-mentioned process is regarded as the progressive failure effect.

Set-out Slide Effect. The set-out slide effect refers to the mechanical process that the slope varies from the static state to the motion one. The set-out slide effect contains two meanings, namely the effect of resistance decrease and the effect of movement.

The effect of resistance decrease. The slope cannot slide immediately, even if the sliding surface comes into being after the progressive failure effect under earthquake forces. It depends on the value of the safety factor which refers to the ratio of the anti-slipping force to the slipping force on the sliding surface of the slope. However, when the earthquake continues, the unsynchronized vibration under the inertia force will come about between the rock mass above the sliding surface and that underneath the sliding surface. On the one hand, it makes the rock mass of the sliding surface crushed and the anti-slipping force reduced. On the other hand, once the relative displacement generates between the two parts of the rock mass, the friction state of rock mass will transform from the state of static friction to the state of kinetic friction. Generally the kinetic friction coefficient of the rock mass is much less than the static friction coefficient, which makes the stable and static rock mass turn into the instable and kinetic one.

The effect of movement. As shown in Fig. 1, the slider moves from point A to point $\mathrm{B}$ under the seismic ground acceleration $a(t)$. This displacement is mainly made up of two parts: the slope displacement $U_{\alpha}(t)$ under the ground acceleration and the relative displacement $U_{\beta}(t)$ between the slider and the sliding surface under the action of the inertial force.

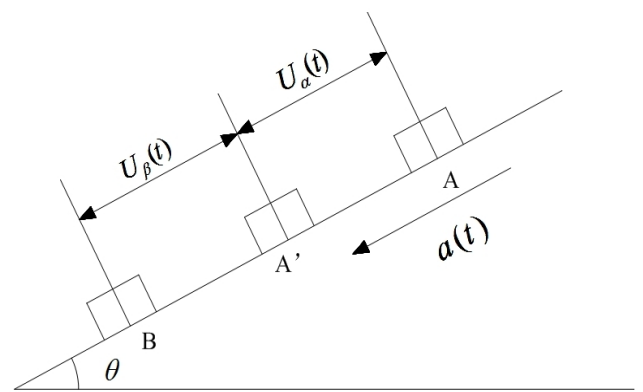

Fig. 1 Schematic of slider movement.

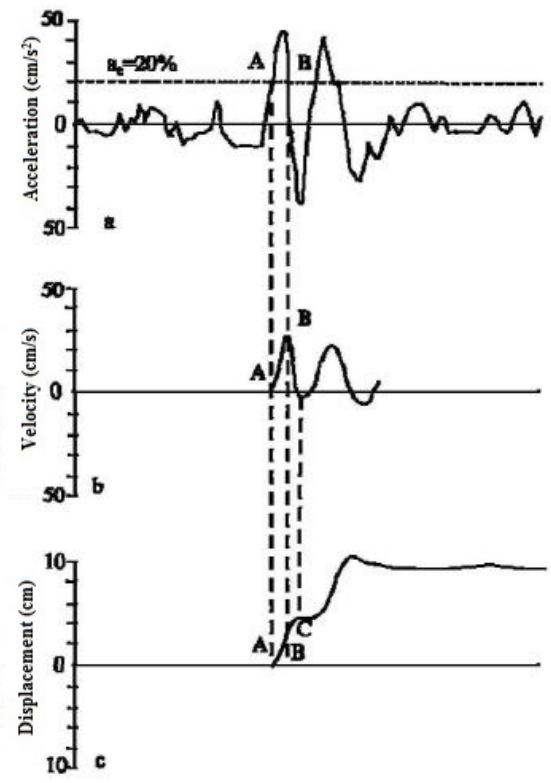

Fig. 2 Schematics of Newmark calculation. (a) Earthquake record and the critical acceleration. (b) Time curve of block velocity. (c) Time curve of block displacement.

Newmark sliding analysis method can be used to calculate the above-mentioned displacement, as shown in Fig. 2. It is set that $a(t)$ represents the seismic ground acceleration function of time and $a_{c}$ represents the critical acceleration. Then the landslide movement can be divided into four stages: the stage of no sliding (from 0 to $t_{a}$ ), the stage of starting sliding $\left(t=t_{a}\right)$, the stage of sliding (from $t_{a}$ to $t_{c}$ ) and the stage of stopping sliding $\left(t=t_{c}\right)$.

When $a(t)<a_{c}$, the slope is stable. 
When $a(t)>a_{c}$ (Point A in Fig. 2), the acceleration $a(t)-a_{c}<0$ is applied on the slope slider which will achieve and ultimately come back to $a_{c}$ (Point B in Fig. 2). At this point, the slider will reach the maximum velocity which can be calculated through the integration of the acceleration curve.

The slider won't stop sliding when arriving at point B ( $\iota \& 0)$, but as a result of $a(t)-a_{c}<0$, the sliding velocity will decrease gradually. The sliding will stop at the time of point $\mathrm{C}$, and the movement will not continue until $a(t)$ exceeds $a_{c}$ again. The displacement from point A to point $\mathrm{C}$ can be obtained via the integration of velocity curve.

The calculation of the block displacement caused by friction is relatively complex. In order to simplify the calculation, it is assumed that the ground acceleration is

$$
a=a_{\max } \sin \omega t
$$

where $a_{\max }$ is the peak ground acceleration and $\omega$ is the circular frequency.

The critical acceleration is set as

$$
a_{c}=\left(F_{S}-1\right) g \sin \theta
$$

where $F_{S}$ is the safety factor, $g$ is the acceleration of gravity and $\theta$ is the slope angle. The calculation formula of the total displacement caused by the horizontal oscillation can be deduced as

$$
U_{h}=\frac{T_{d} a_{c}}{6 \pi \omega}\left[2\left(\pi-2 \arcsin \frac{a_{c}}{a_{\max }}\right) \cdot \sqrt{\frac{a_{\max }^{2}}{a_{c}^{2}}-1}\left(\pi-2 \arcsin \frac{a_{c}}{a_{\max }}\right)^{2}\right]
$$

The acceleration effect. If the calculated result of the slope displacement is only a few millimeters through Newmark analysis method, the slope can be completely stable after earthquake even if the peak ground acceleration exceeds the critical acceleration. If the result is more than dozens of centimeters or a few meters, a massive landslide will occur under the seismic forces. The landslide starting speed can be calculated by

$$
V_{v o}=\sqrt{\frac{T_{d}}{3 \pi \omega}} \cdot a_{c}\left(\sqrt{\frac{a_{\max }^{2}}{a_{c}^{2}}-1}+\arcsin \frac{a_{c}}{a_{\max }}-\frac{\pi}{2}\right)
$$

According to the formula above, the landslide starting speed is proportional to the square root of the earthquake duration $T_{d}$, and inversely proportional to the square root of the circular frequency $\omega$. Namely the starting speed will be higher with the growth of earthquake duration and the period of vibration. The starting speed is still associated with the peak ground acceleration $a_{\max }$. A large-scale and high-speed landslide can be caused more easily if the peak ground acceleration $a_{\max }$ is greater. In addition, the starting speed is also affected by the state of the slope stability. The speed will be much higher if the slope stability approaches its critical state.

\section{Numerical Simulation of Dynamic Instability Failure Mechanism}

According to the above-mentioned analysis, the dynamic failure process of the CRS is a complex mechanical process with the transition from quantitative change to qualitative change and from static state to kinetic state. In general, the accumulation process of quantitative change is a small deformation process, which is usually simulated through finite element method (FEM). And the process of qualitative change belongs to large deformation process, which is usually simulated through discrete element method (DEM). FLAC3D possesses the characteristics of both FEM and DEM; therefore, the instability failure mechanism of the CRS can be realistically reflected by using 
FLAC3D. In this section, FLAC3D is utilized to simulate and analyze the instability process and failure mechanism of the CRS.

Dynamic Numerical Analysis Model. The dimension and material groups of the calculation model are shown in Fig. 3. The static constitutive model and material parameters can be adopted to solve the dynamic issues by using FLAC3D. Moore-Coulomb model is used and the corresponding material parameters are shown in Table 1. In the dynamic calculation, the static boundary condition is applied at the bottom of the model, and the free-field boundary condition is used to simulate the state of infinite space around the slope model. Four monitoring points are set up to measure the dynamic responses. Monitoring points from No. 1 to No. 3 are respectively arranged at the top, the middle and the foot of the slope surface. The point of No. 4 is located at the bottom of the model to measure the primary seismic waves.

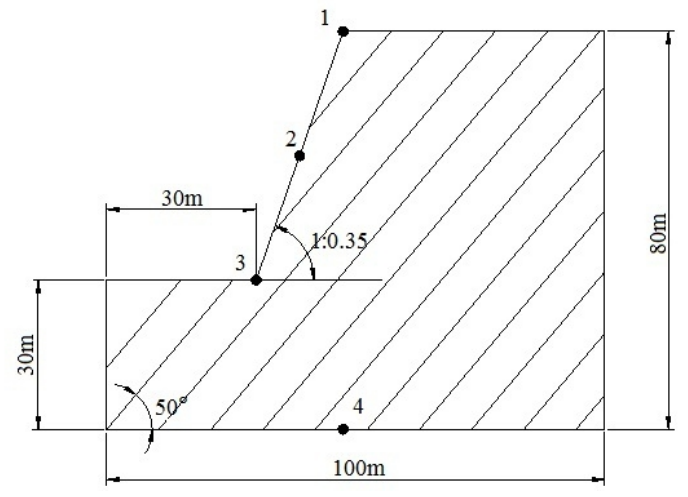

(a)

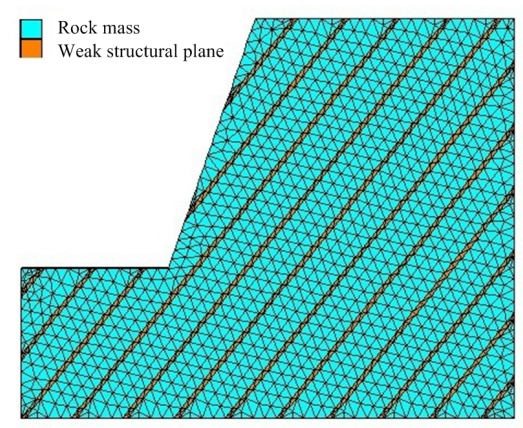

(b)

Fig. 3 Dynamic calculation model. (a) The dimension. (b) The material groups.

Table 1 Material parameters of numerical calculation model.

\begin{tabular}{|c|c|c|c|c|c|c|}
\hline & $\begin{array}{c}\text { Bulk } \\
\text { Modulus } \\
(\mathrm{GPa})\end{array}$ & $\begin{array}{c}\text { Shear } \\
\text { Modulus } \\
(\mathrm{GPa})\end{array}$ & $\begin{array}{c}\text { Cohesion } \\
(\mathrm{MPa})\end{array}$ & $\begin{array}{c}\text { Internal } \\
\text { Friction } \\
\text { Angle }\left({ }^{\circ}\right)\end{array}$ & $\begin{array}{c}\text { Tensile } \\
\text { Strength } \\
(\mathrm{MPa})\end{array}$ & $\begin{array}{c}\text { Density } \\
\left(\mathrm{kg} / \mathrm{m}^{3}\right)\end{array}$ \\
\hline Rock mass & 1 & 0.6 & 0.6 & 38 & 0.5 & 2500 \\
\hline Weak structural plane & 0.196 & 0.0752 & 0.08 & 27 & 0.09 & 2000 \\
\hline
\end{tabular}

The measured Taft acceleration time history curve is applied as the seismic ground motion, of which the duration is $20 \mathrm{~s}$ and the peak acceleration is $70 \mathrm{~cm} / \mathrm{s}^{2}$, as shown in Fig. 4.

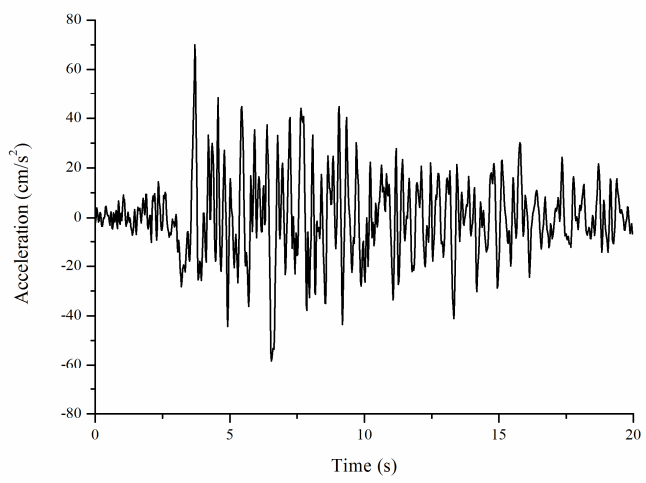

Fig. 4 Taft acceleration time history curve. 
Numerical Analysis Results of Instability Failure Mechanism. The dynamic analysis is carried on with the above-mentioned earthquake acceleration time history curve applied to the slope model. The time history curves of displacement in the $\mathrm{x}$ direction is shown in Fig. 5(a), and the relative displacement between monitoring points from No. 1 to No.3 and the reference point (No. 4) is shown in Fig. 5(b). The displacement time history curves of the former 3 monitoring points are underneath the curve of No. 4. It shows that the slope body has different degrees of relative displacement under earthquake forces. Among the displacement time history curves, the curve of No. 3 has the similar variation tendency with No. 4, and they keep small relative displacement until the end of the earthquake. This result indicates that the rock mass at the foot of the slope does not generate excessive relative sliding. After the earthquake, the rock around the slope foot is stilly stable.

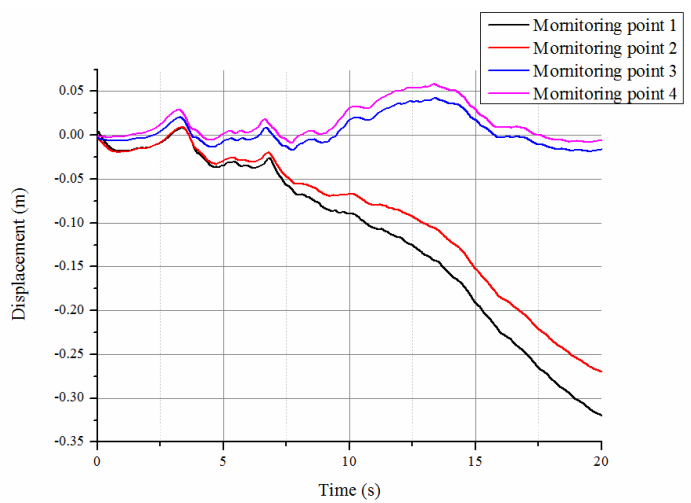

(a)

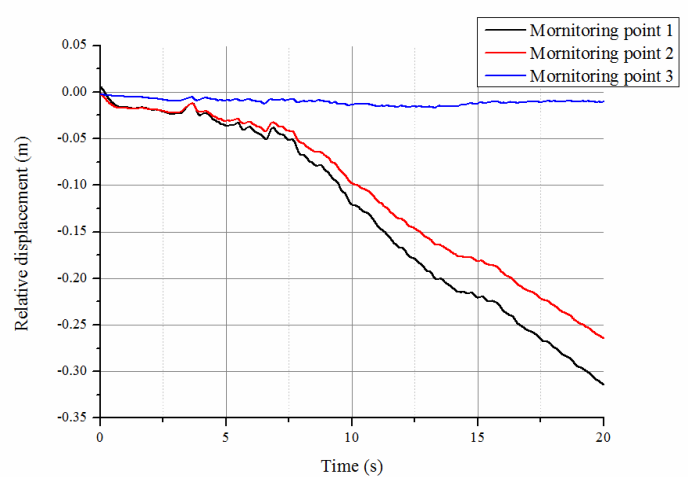

(b)

Fig. 5 Displacement time history curves of monitoring points. (a) The displacement curves in the x direction. (b) The relative displacement curves in the $\mathrm{x}$ direction.

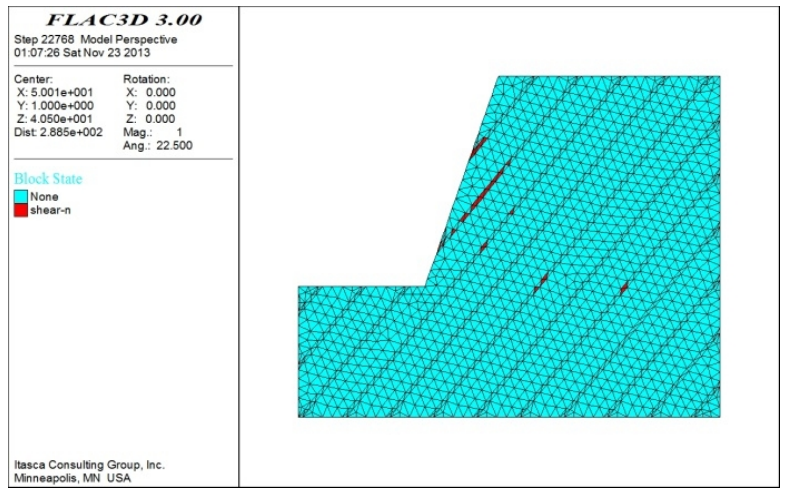

(a) $t=2 s$

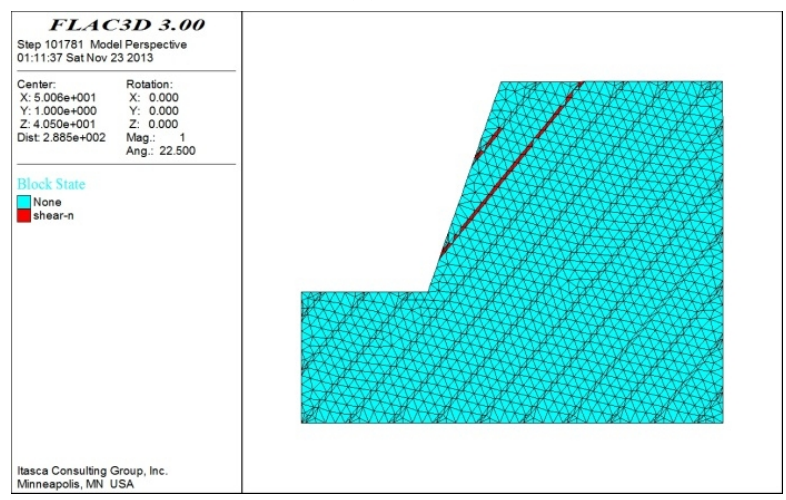

(c) $t=6 s$

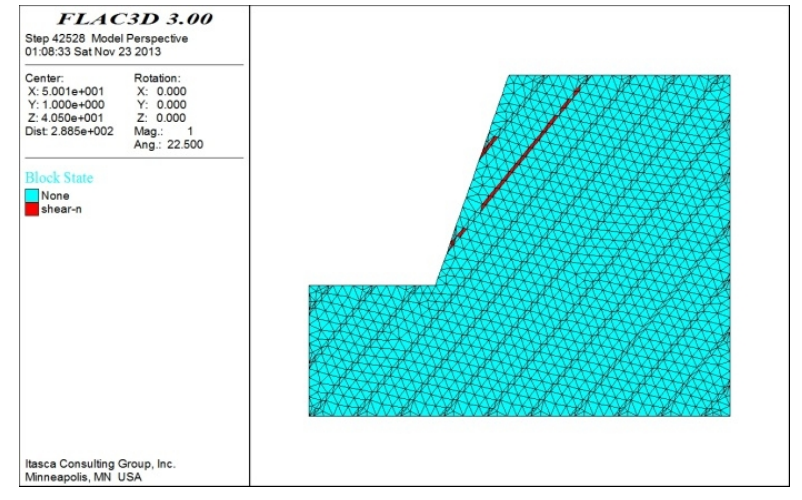

(b) $\mathrm{t}=4 \mathrm{~s}$

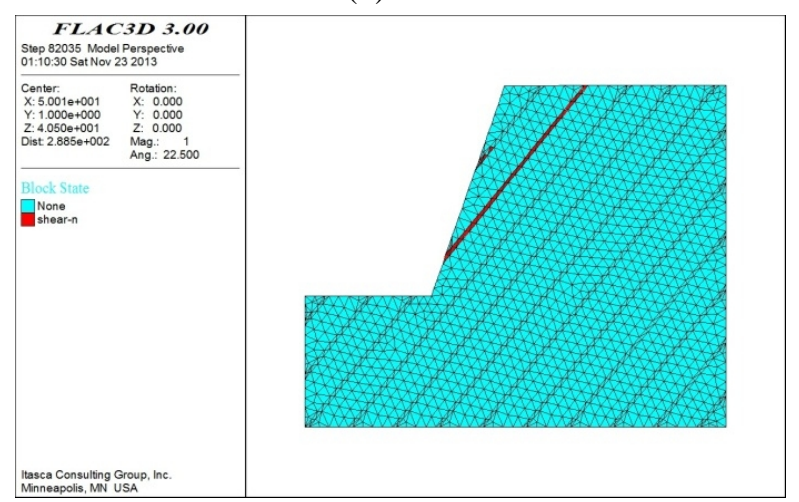

(d) $t=8 \mathrm{~s}$

Fig. 6 Gradual development of plastic area on sliding surface. 
According to the displacement curves of point No.1 and point No.2, at the beginning of the earthquake, both them has the similar displacement variation tendency with the reference point, meanwhile their relative displacement also remains little difference. When the earthquake lasts for about $8 \mathrm{~s}$, the relative horizontal displacement of point No.1 and point No.2 has reached $5 \mathrm{~cm}$. At this moment, the plastic area of the slope sliding plane are completely connected from the bottom to the top (as shown in Fig. 6), which marks the end of the progressive failure effect.

Fig. 7 shows the velocity time history curve of monitoring point No.1 at the top of the slope model. Associated with the former displacement curve of the same point in Fig. 5(a), after the progressive failure effect, the displacement time history curve presents a downward trend when the function time of the earthquake force is between $8 \mathrm{~s}$ and $11.26 \mathrm{~s}$. However, the velocity time history curve still fluctuates around the zero line, that is to say the landslide mass still has the movement in the opposite direction from the slip direction of the slope. During this time, the slope is in the stage of the set-out slide effect.

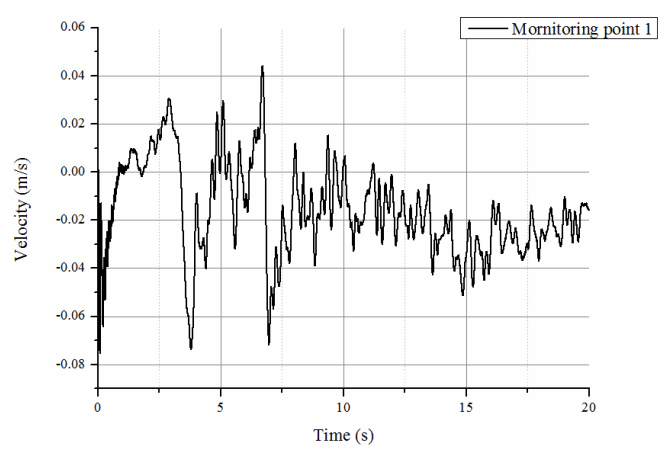

Fig. 7 Velocity for monitoring point No.1.

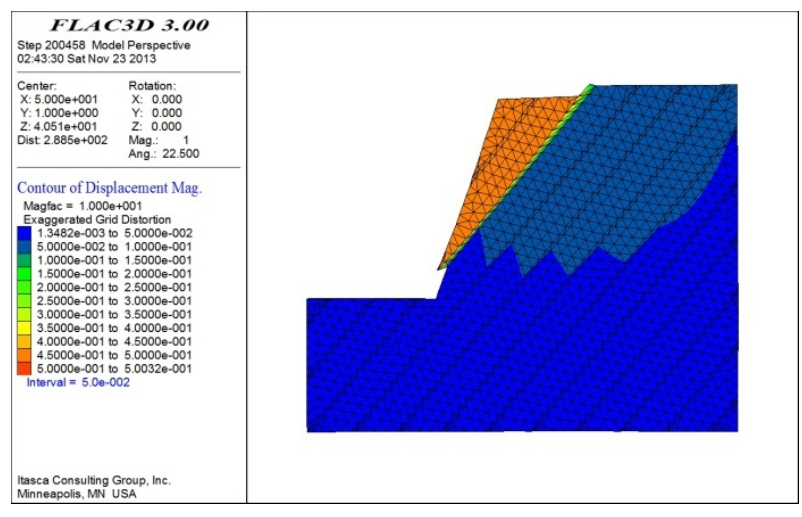

Fig. 8 Slope displacement nephogram at the end of earthquake.

After $11.26 \mathrm{~s}$, the landslide mass only generates displacement along the slip direction, which indicates the beginning of the landslide. At this moment the slope is in the stage of accelerating effect. The displacement nephogram of the slope model at the end of earthquake is shown in Fig. 8. It can be seen that the slope has generated shear slip failure, and the maximal displacement has reached $50 \mathrm{~cm}$.

\section{Dynamic Stability Safety Evaluation of Consequent Rock Slope}

Instability Criterion of Dynamic Strength Reduction Method. Strength reduction method can be applied to the dynamic stability analysis of the CRS. In the dynamic strength reduction method (DSRM), the shear strength indexes are reduced continuously, and the dynamic time history analysis of CRSs with different shear strength is carried on, respectively. When the slope reaches the state of the critical destruction, the corresponding reduction factor is defined as the slope dynamic safety factor. Therefore, whether the dynamic critical state is appropriate will directly restrict the reasonability of the result of dynamic stability safety evaluation. A scientific and reasonable instability criterion must be proposed anyway.

The biggest characteristic of the dynamic instability failure mechanism of consequent rock slope is the motion variations of the landslide mass, namely the variations from small deformation to large deformation and from static state to kinetic state. The progressive failure effect is a small deformation process, while the set-out slide effect and the acceleration effect are large deformation processes. And the set-out slide effect and the acceleration effect are the signs of landslide under earthquake. The instability failure of slope may not occur if only the progressive failure effect is 
finished. Therefore, it can be regarded that whether the set-out slide effect and the acceleration effect take place as the dynamic instability criterion of consequent rock slope. The permanent displacement generated in the set-out slide effect and the acceleration effect is much greater than that generated in the progressive failure effect. Therefore, the specific form of the criterion above is the mutability of the permanent displacement.

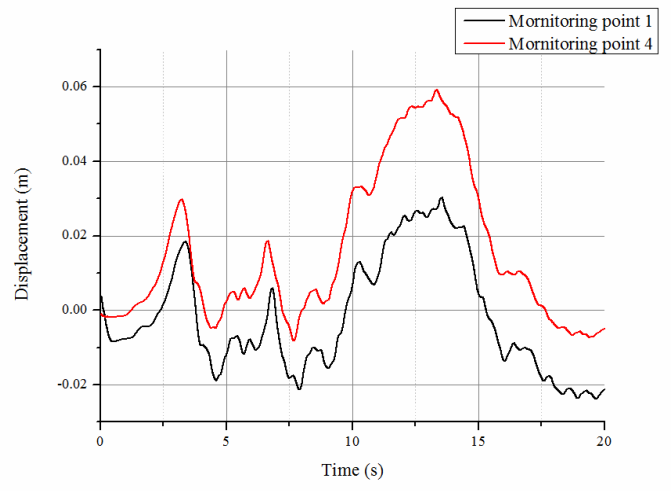

(a)

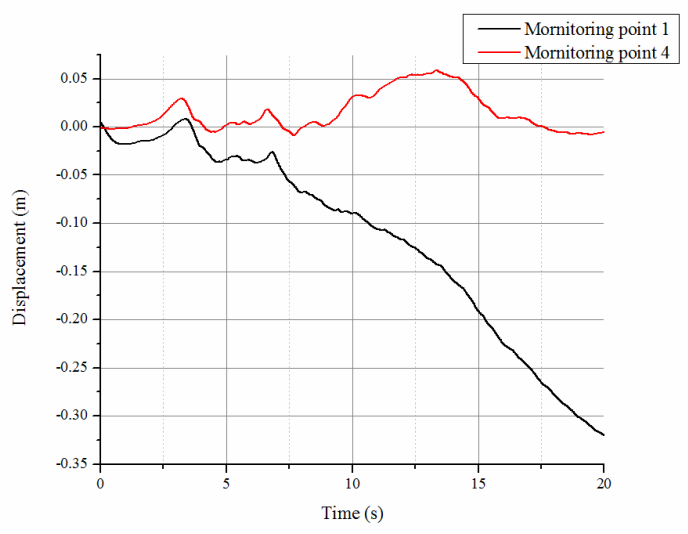

(c)

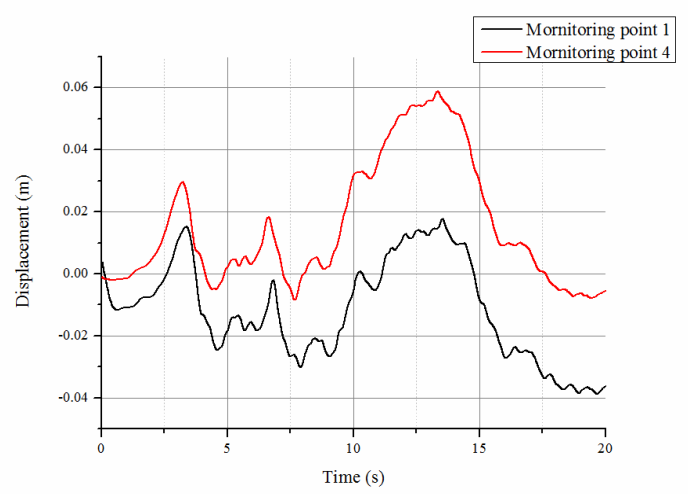

(b)

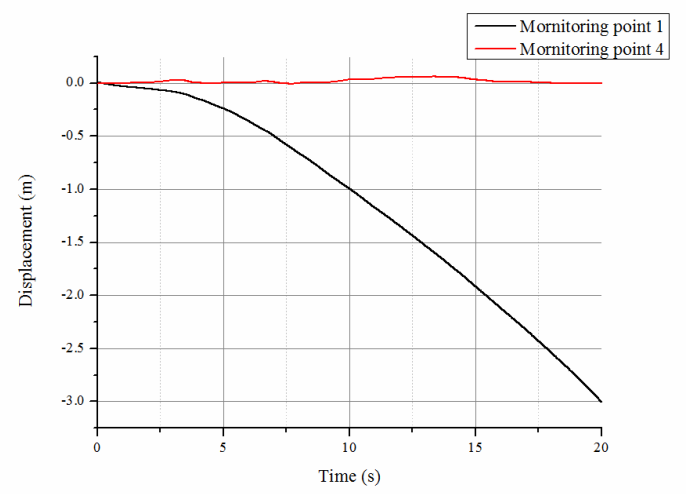

(d)

Fig. 9 Displacement time history curve of the monitoring point 1 changing along with the dynamic reduction factors. (a) $K_{S}=0.94$. (b) $K_{S}=0.95$. (c) $K_{S}=0.96$. (d) $K_{S}=0.97$.

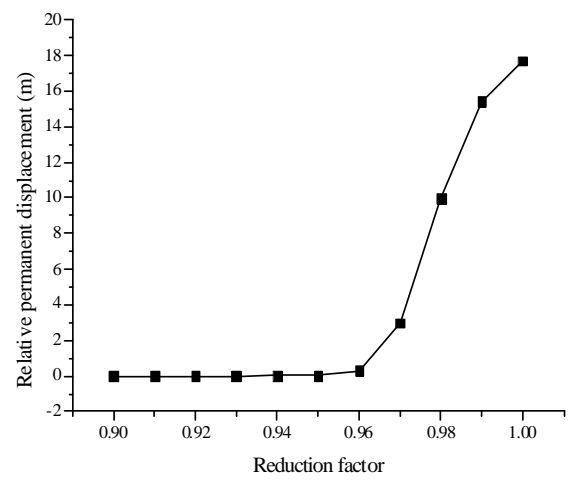

Fig. 10 Relative permanent displacement vs. reduction factor curve.

The displacement time history curves of monitoring point No.1 changing along with the dynamic reduction factors are shown in Fig. 9. When $K_{S}$ (the dynamic reduction factor) equals 0.94 , the horizontal relative permanent displacement on the top of slope is $0.021 \mathrm{~m}$ by the end of the 
earthquake. When $K_{S}$ equals 0.95 , the permanent displacement will increase to $0.036 \mathrm{~m}$. When $K_{S}$ equals 0.96 , the permanent displacement will increase rapidly to $0.314 \mathrm{~m}$ due to the set-out slide effect and the acceleration effect. When $K_{S}$ achieves 0.97 , the progressive failure effect is completed in a very short time, and the permanent displacement of monitoring point No.1 finally reaches 3.004 $\mathrm{m}$. The Fig. 10 shows how the relative permanent displacement changes along with reduction factors. It is obvious that the curve starts to mutate at the point when $K_{S}$ equals 0.96 . Therefore, the dynamic safety factor of the consequent rock slope can be determined as 0.96 .

Results of Dynamic Stability Safety Evaluation. Four seismic wave records are selected to evaluate the dynamic stability of the slope model, including: (1) Cape Mendocino seismic wave, recorded in 1992 with the predominant period of 0.26s; (2) Taft seismic wave, recorded on July 21, 1952 with the predominant period of 0.44s; (3) EL Centro earthquake wave, recorded in 1940 with the predominant period of 0.56s; (4) Tianjin seismic wave, recorded on November 25, 1976 with the predominant period of $1.04 \mathrm{~s}$. The baseline correction and filtering to these seismic waves must be finished before input. Dynamic strength reduction method is used to evaluate the dynamic stability of consequent rock slope. The curves of relative permanent displacement vs. reduction factor are shown in Fig. 11.

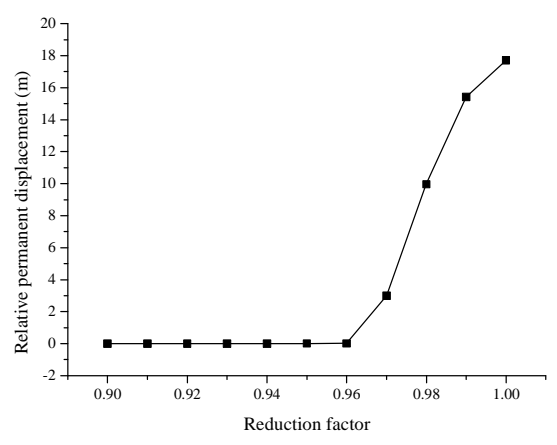

(a)

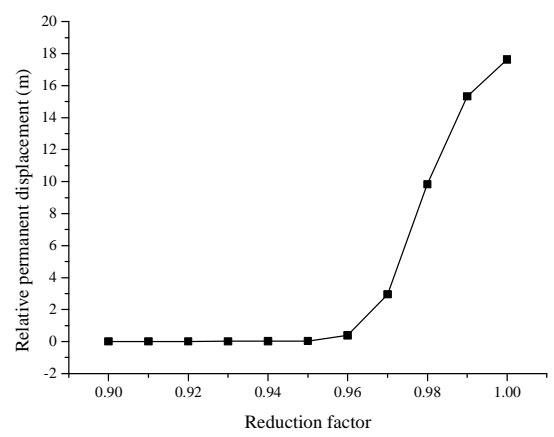

(c)

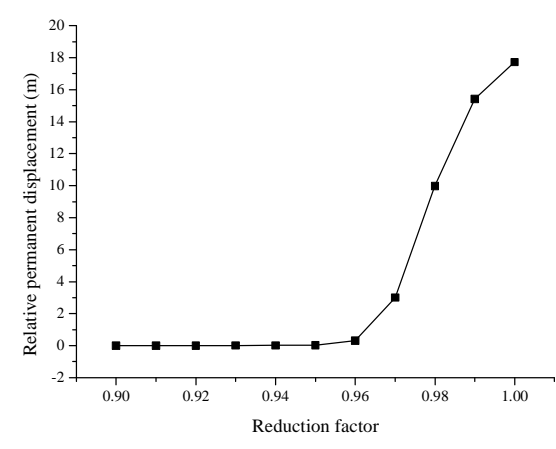

(b)

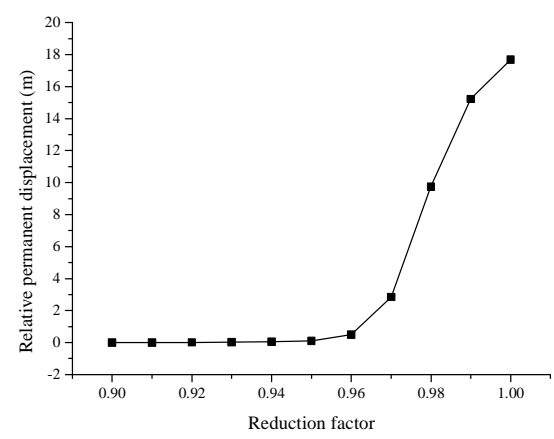

(d)

Fig. 11 Relative permanent displacement vs. reduction factor under various earthquakes. (a) Cape Mendocino wave. (b) Taft wave. c) EL Centro wave. (d) Tianjin wave

As shown in Fig. 11, the four relative permanent displacement curves are similar, which begins to mutate when $K_{S}$ achieves 0.96 . The dynamic stability safety factor of the consequent rock slope under frequent earthquake can be determined as 0.96 . The consequent rock slope cannot meet the requirement of the dynamic stability. Necessary measures must be taken to reinforce the slope.

The natural period of the slope is $0.39 \mathrm{~s}$ according to the results of the dynamic characteristics test of the numerical model. The results in Fig. 11 show that the ground predominant period cannot 
influence the result of the dynamic safety factor obviously.

\section{Summary}

So far, the slope dynamic stability problem is bothering in geotechnical engineering field. The study about the instability failure mechanism and stability analysis of the consequent rock slope is still in the exploratory stage. The scholars at home and aboard still fail to reach a unified understanding. The research work of this paper is focused on the dynamic stability problem of consequent rock slope. The main research conclusions include:

(1) Based on massive research, the instability failure mode of consequent rock slope is highly summarized into three effects by $\mathrm{Mao}$ and $\mathrm{Hu}$ : the progressive failure effect, set-out slide effect and acceleration effect. On this basis, the numerical analysis of instability failure mechanism of the consequent rock slope is conducted in this paper. The analysis results show the progressive failure process of the slope from quantitative change to qualitative one and from static stage to kinematic stage under the oscillation of seismic wave.

(2) According to the dynamic instability failure characteristics of consequent rock slope, the instability criterion of the dynamic strength reduction method is proposed. It is regarded that whether the set-out slide effect and the acceleration effect take place as the dynamic instability criterion of consequent rock slope in this paper. Dynamic strength reduction method is adopted to evaluate the dynamic stability of consequent rock slope under the frequent earthquake. Based on the comparison of the analysis results of consequent rock slope under four kinds of seismic waves, it can be concluded that the dynamic stability of slope is not sensitive to the ground predominant period.

\section{Acknowledgement}

This work was financially supported by the National Key Research and Development Program of China (SQ2017YFC150201), the Natural Science Foundation of Liaoning Province of China (2015020595), and Science Foundation of Liaoning Education Department (LJZ2016029).

\section{Literature References}

[1] J.J. Chen: The Analysis of Seismic Dynamic Response of Slopes in Mountains with Complex Geological Backgrounds, Master Dissertation, Chengdu University of Technology, Chengdu, 2009. (in Chinese)

[2] L.P. Liu, S.J. Yang and Y.M. Li: Journal of Chongqing University (Natural Science Edition), 30(5) (2007), p. 31. (in Chinese)

[3] Y.M. Men, J.B. Peng, X.C. Li and J.B. Hao: World Earthquake Engineering, 20(4) (2004), p. 131. (in Chinese)

[4] O.C. Zienkiewicz, C. Humpheson and R.W. Lewis: Geotechnique Vol. 25 (1975), p.671.

[5] Y.M. Chen and D.P. Xu: FLAC/FLAC3D Foundation and Practical Engineering (China Water Conservancy and Hydropower Press, Beijing China 2008). (in Chinese)

[6] Y.L. Mao, G.T. Hu, F.S. Zhao and X.H. Mao: Journal of Xi'an Engineering University Vol. 20 (1998), p. 45. (in Chinese)

[7] Y.L. Mao, G.T. Hu, X.H. Mao and Y.W. Shi: Journal of Engineering Geology Vol. 9 (2001), p. 74. (in Chinese) 